\title{
NFATc1 is Suppressed in Tumor Microenvironment of Hodgkin Lymphoma
}

\author{
Krisna Murti ${ }^{1 *}$, Neti Neti ${ }^{1}$, Nyiayu Fauziah Kurniawati ${ }^{1}$, Ika Kartika ${ }^{1}$, Riana \\ Sari Puspita Rasyid ${ }^{2}$, Zen Hafy ${ }^{2}$
}

\begin{abstract}
Objective: The aims of this research are to evaluate the expression and distribution of NFATc1 in tumor microenvironment of Hodgkin lymphoma. Methods: Twenty-eight cases of Hodgkin lymphoma were selected. Clinicopathological data of age, gender, location and subtypes were obtained. Immunohistochemistry was performed to the all cases by using anti-CD163, anti-NFATc1 and anti-PD-L1 antibodies. All protein expression was calculated by using Image J software. Results: Nuclear expression of NFATC1 was not observed in Hodgkin cells neither in TAM nor in small lymphocytes surrounding Hodgkin cells in all the samples, this meant that NFATc1 showed negative nuclear expression in almost all these cells. Cytoplasmic expression of NFATc1 was observed in small lymphocytes surrounding tumor cells. While there were only few small lymphocytes which were located far from tumor cells showed nuclear expression of NFATC1. Meanwhile, 57.14\% samples showed high density of TAMs CD163+, and 50\% tumor cells as well as 50\% TAMs exhibited positive $P D-L 1$ expression. In addition, all macrophages did not have NFATc1 expression both in their nuclei and in their cytoplasm. Conclusion: NFATcl was suppressed both in Hodgkin cells and inflammatory cells surrounding the tumor cells. This condition may contribute to progressivity and aggressiveness of the diseases. Therefore, certain mechanisms to reactivate functional NFATcl in HL tumor microenvironment may be necessary; hence, the tumor cells are able to be eradicated by patient's immune mechanisms.
\end{abstract}

Keywords: Hodgkin lymphoma- tumor microenvironment- NFATc1-CD163-PD-L1

Asian Pac J Cancer Prev, 22 (6), 1943-1948

\section{Introduction}

From early development to tumor progression and then metastasis, tumor cells are counter acted by numerous types of tumor microenvironment (TME) elements i.e., stromal factors and immune cells. Therefore, beside the other factors, TME is also an essential component in determinating tumor behavior and prognosis (Kim and Bae, 2016). Numerous markers and therapeutic strategies were developed based on TME context.

In 2018, Hodgkin lymphoma (HL) incidence of new cases were around 79,990 with number of deaths were circa 26,167 (Bray et al., 2018). The incidence of HL varies considerably by age, sex, ethnicity, geographic location and socioeconomic status, and its rates are higher among males and in developed countries, but lower in Asian population. Meanwhile, mortality rates were lower in underdeveloped and higher developing regions (Zhou et al., 2019; Salati e al., 2014). Indonesia ranks $25^{\text {th }}$ in incidence of HL (Ferlay, 2013). Young population at ages 15 to 25 years are mostly affecting by HL with higher incidence (Bigenwald et al., 2017). Despite its relatively low incidence and its low lifetime risk, HL comprises
$15 \%$ of all cancers in young adults with a high impact on quality of life (Salati et al., 2014).

HL is a curable disease; more than $90 \%$ cure rate for patients with early disease and in more than $70 \%$ patients with advanced disease (Shanbhag and Ambinder, 2018). The crucial point is to recognize high-risk patients who will relapse after initial therapy. Therefore, identifying these high risks patients by characterization of pathobiological and clinical prognostic factors then followed by designing properly novel treatment strategies with minimal treatment toxicities is demanding.

Morphologic characteristic of HL is heavily infiltrating inflammatory cells surrounding tumor cells as its tumor microenvironment (Calabretta et al., 2019). In classical $\mathrm{HL}$ (cHL) cells NF-KB is constitutively activated (Weniger and Küppers, 2016), however the exact factors regulate its microenvironment is still unclear. Latest findings revealed that abundant component cellular and humoral generated by interaction of Hodgkin cells with their environment, which might contribute to the characteristic background inflammatory cells (Calabretta et al., 2019).

Macrophages are the other types of inflammatory cells observed heavily infiltrate the background of Hodgkin 
cells. Unlike $P D-L 1$ expressed on tumor cells, $P D-L 1$ expressed on macrophages is able to protect macrophages from destruction by T cells (Singhal et al., 2019). In addition to this, other studies showed that $P D-L 1$ in macrophages inducing T cell anergy and M2 polarization (Lu et al., 2019).

Known as an essential transcription factor in many physiologic systems comprising immune cells (Vaeth and Feske, 2018), including in regulation of PD-1 activation (Oestreich et al., 2008), nuclear factor of activated T cell (NFATc1) has roles in tumor microenvironment (Li et al., 2018; Gholami et al., 2017). NFATc1 may contribute to the molecular pathways entailed in tumor microenvironment of HL, which, then both promote to HL progression and worsen prognosis.

The aims of this research are to evaluate the expression and distribution of NFATCl in tumor microenvironment of Hodgkin lymphoma. Together our results may identify NFATCl as promising target for alternative novel marker of prognostic and or predictive factors of Hodgkin lymphoma.

\section{Materials and Methods}

\section{Patient data}

Initially, we collected 44 cases of Hodgkin lymphoma diagnosed based on the 2016 World Health Organization classification (Swerdlow et al., 2017) from January 2014 to November 2019 at Department of Anatomic Pathology, Faculty of Medicine University of Sriwijaya, Dr. Mohammad Hoesin Hospital, Palembang, Indonesia. After careful selection based on quality of fixation and processing which can be assessed by carefully examined the HE and IHC slides, 28 cases were obtained as samples. Clinicopathological parameters i.e., age, gender of patients, subtypes, and location of tumors were attained from patient's pathology records. Ethical committee approval from Faculty of Medicine University of Sriwijaya was also attained.

\section{Immunohistochemical analysis}

The paraffin blocks of selected HL cases were retrieved from the archives. Immunohistochemical staining was conducted using manual system according to standard immunohistochemical protocol of our lab. The analyses were validated using appropriate negative and positive controls by using several tissue blocks consisting of tonsil, appendix, melanoma and breast cancer tissues. After sectioning, the blocks were dried in a lab heating and drying followed by deparaffinization and rehydration. Then antigen retrieval was performed by treating the slides in a microwave in citrate buffer. After blocking step the tissues were incubated for 60 minutes with primary antibody NFATc1 (clone 7A6, dilution 1:200, BD Pharmingen, Franklin Lakes, New Jersey), CD163 (clone 10D6, rabbit, monoclonal, dilution 1:100, thermo fisher, USA) and PD-L1 (clone SP142, dilution 1:100, Abcam, Cambridge, MA). Lastly, the slides were covered with mounting medium and coverslips. Stained tissues and all pictures were analyzed and captured using Olympus BX41 (Tokyo, Japan) couple with camera (12MP1/1.7" Sony
Exmor CMOS Sensor, Beta Industrial Digital Camera, China) at a $\times 400$ magnification.

\section{Expression of NFATc1, CD163, and PD-L1}

The positive expression of all antibodies was determined disregard staining intensity, since the later was most likely influenced by inconsistency of tissue fixation and processing. NFATCl positive expression was determined in nuclei of tumor cells as well as in lymphocytes and macrophages surrounding tumors. Positive expression of CD163 was calculated in membrane and or cytoplasm of macrophages around tumor cells. In addition, positive expression of $P D-L 1$ was counted in membrane of Hodgkin tumor cells and macrophages around tumor cells. Image $\mathrm{J}$ was used to quantify the numbers of protein expression of NFATC1, CD163, and PD-L1.

\section{Density of NFATc1, CD163, and PD-L1}

Reactivity of every antibody was differentiated into high and low density based on cut-off point obtained from median value. At the beginning the most concentrated five locations containing brown staining either NFATC1, or $C D 163$ or $P D-L 1$ were selected under low power field $(100 x)$. Then among these areas, the five most densest focuses were carefully chosen and photographed under high magnification (400x). By using image $\mathrm{J}$ software, the all cells expressed either $N A F T c 1$, or $C D 163$, or $P D-L 1$ were calculated and noted. Of these five areas, the average was counting by using excel. The median of all samples of each antibody was considered as a cut-off point for differentiation of $N A F T c 1$, or $C D 163$, or $P D-L 1$ expression into high or low density.

\section{Statistical Analysis}

Since NFATC1 expression was negative in the evaluated area of all the samples, the statistical analysis was not performed.

\section{Results}

\section{Patients Characteristics}

Among 28 total samples, our data only have one case of NLPHL and $27 \mathrm{cHLs}$. The age was differentiated into five groups i.e., under 20 years $(10.7 \%)$, between 20 to 29 years $(25 \%)$, between 30 to 39 years $(10.7 \%)$, between 40 to 49 years $(28.6 \%)$ and after 50 years $(25 \%)$. More patients in the ages of 40 to 49 years suffer from HL. Males suffer from HL more than that in females $(57.1 \%)$. Tumor masses were mostly found in head and neck (78.6\%). Lymphocyte-rich cHL was the subtype which mostly observed (57.1\%) among others (Table 1).

\section{Immunohistochemistry \\ NFATc1, CD163 and PD-L1}

Nuclear expression of NFATc1 was not observed in Hodgkin cells neither in TAM nor in small lymphocytes surrounding Hodgkin cells in all samples (Table 2), this meant that NFATCl showed negative expression in almost all these cells. There were only few small lymphocytes showed nuclear expression of NFATC1 in some patients 


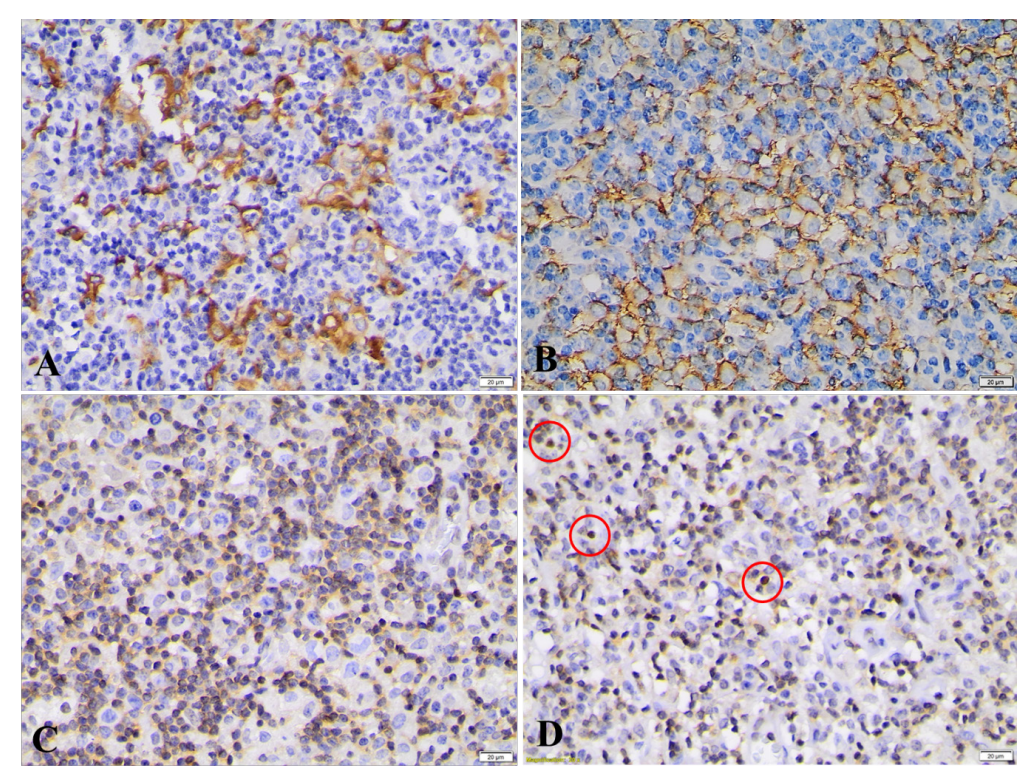

Figure 1. The Immunoreactivity of CD163, PD-L1 and NFATc1 Proteins of Patient \#1. A. Showed various positive cytoplasmic expression of TAMs CD163+. B. Immunoreactivity of PD-L1 in membrane of Hodgkin cells and TAMs. C. Demonstrated various negative nuclear expression of NFATc1 protein in all cells; the only finding was cytoplasmic expression of NFATc1, particularly in small lymphocytes surrounding the Hodgkin cells. D. It can be seen few small lymphocytes showed nuclear expression but far from Hodgkin tumor cells (red circles). Original magnifications $\times 400$.

Table 1. Patient Characteristics

\begin{tabular}{|c|c|c|}
\hline Clinical features & $\mathrm{N}(28)$ & $\%$ \\
\hline \multicolumn{3}{|l|}{ Age (years) } \\
\hline$<20$ years & 3 & 10.7 \\
\hline $20-29$ & 7 & 25.0 \\
\hline $30-39$ & 3 & 10.7 \\
\hline $40-49$ & 8 & 28.6 \\
\hline$\geq 50$ years & 7 & 25.0 \\
\hline \multicolumn{3}{|l|}{ Gender } \\
\hline Male & 16 & 57.1 \\
\hline Female & 12 & 42.9 \\
\hline \multicolumn{3}{|l|}{ Location } \\
\hline Head-neck & 22 & 78.6 \\
\hline Body & 2 & 7.1 \\
\hline Extremities & 4 & 14.3 \\
\hline \multicolumn{3}{|c|}{ Subtypes and Variant } \\
\hline NLPHL & 1 & 3.6 \\
\hline \multicolumn{3}{|l|}{ CHL } \\
\hline NSCHL & 4 & 14.3 \\
\hline LRCHL & 16 & 57.1 \\
\hline MCCHL & 7 & 25.0 \\
\hline LDCHL & 0 & 0.0 \\
\hline
\end{tabular}

(Figure 1). These cells were located far from tumor cells, while small lymphocytes surrounding tumor cells have only cytoplasmic expression of NFATc1 (Figure 1 and Figure 2). Approximately 57.14\% samples showed high density of TAMs CD163+. In addition, all macrophages did not have NFATC 1 expression both in their nuclei and in their cytoplasm's. The expression of $P D-L 1$ was observed in tumor cells and in TAMs surrounding tumor cells, with similar percentage $(50 \%)$ both in high and low density in those two types of cells (Table 2).

\section{Discussion}

Recent studies have identified the impact of non-neoplastic cells on disease pathobiology, particularly immunohistochemical studies of cells in the tumor microenvironment. As a result, some biomarkers have identified and translated into clinical practice. The transcription factors NF- $\mathrm{KB}$ and NFAT are known as essential factors in activation of B cell lymphocytes (Muhammad K et all., 2014). However, in Hodgkin cells NFATc1 is not expressed caused by epigenetic silenced mechanism (Akimzhanov et al., 2008), while NF- $\kappa B$ is constitutively active in these tumor cells (Weniger and Kuffer, 2016). Our finding confirmed the results of previous studies (Akimzhanov et al., 2008; Marafioti et al.,

Table 2. The Expression of NFATc1, CD163 and PD-L1

\begin{tabular}{lcccccc}
\hline Antibodies & \multicolumn{2}{c}{ Lymphocytes } & \multicolumn{2}{c}{$\mathrm{M} \Phi$} & \multicolumn{2}{c}{ Tumor cells } \\
& $\mathrm{H}$ & $\mathrm{L}$ & $\mathrm{H}$ & $\mathrm{L}$ & $\mathrm{H}$ & $\mathrm{L}$ \\
\hline NFATc1 & 0 & $0(0 \%)$ & 0 & 0 & 0 & 0 \\
& $0 \%$ & & $0 \%$ & $0 \%$ & $0 \%$ & $0 \%$ \\
CD163 & - & - & $16(57.14 \%)$ & $12(42.86 \%)$ & - & - \\
PD-L1 & - & - & $14(50 \%)$ & $14(50 \%)$ & $14(50 \%)$ & $14(50 \%)$ \\
\hline
\end{tabular}

$\mathrm{N}, 28 ; \mathrm{M} \Phi$, macrophages; H, high; L, low 


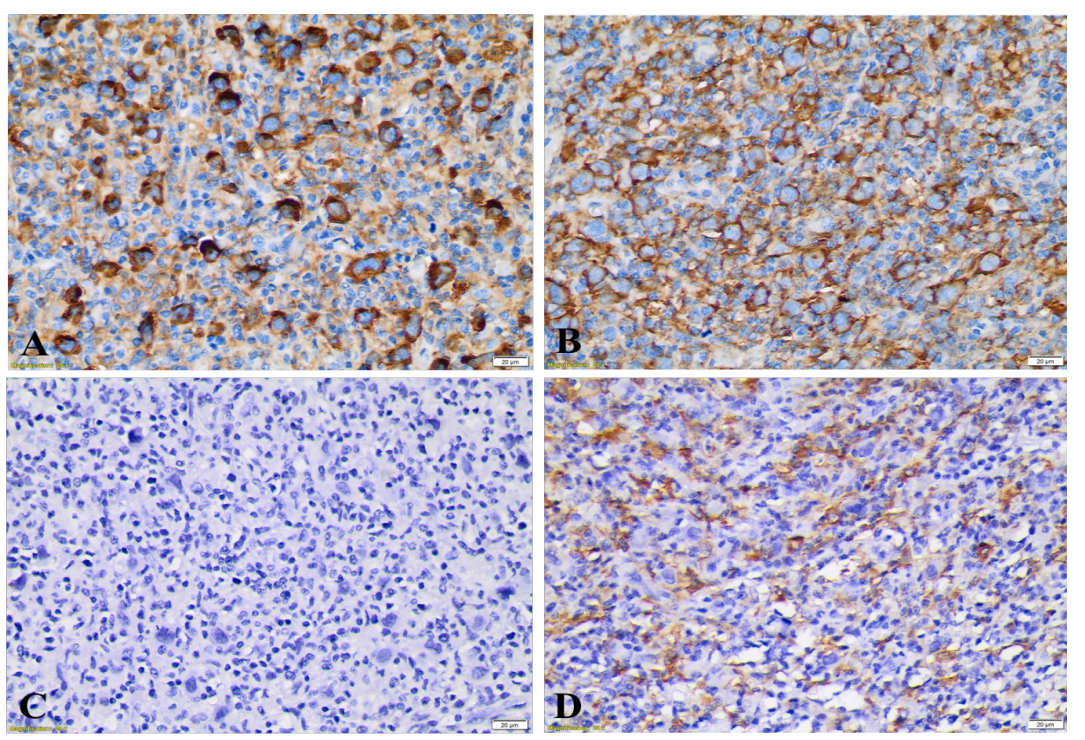

Figure 2. The Immunoreactivity of CD163, PD-L1 and NFATc1 Proteins of Patient \#2. A. Varied immunoreactivity of PD-L1 in membrane of Hodgkin cells and TAMs. B. Positive cytoplasmic expression of TAMs CD163+. C. Negative nuclear expression of NFATc1 protein in all cells. D. Membrane expression of CD163. Original magnifications $\times 400$.

2005) that NFATC1 was not expressed in Hodgkin cells. However, NFATCl expression in tumor microenvironment was not discussed in earlier experiments. Our data showed that there were only few small lymphocytes expressed nuclear NFATC1, but these cells were situated far from tumor cells. While small lymphocytes which located closed to Hodgkin cells only showed cytoplasmic NFATc1 expression, none of them have NFATC1 nuclear expression.

It is known that $\mathrm{T}$ cell lymphocytes surrounding Hodgkin cells exhibited unusual phenotypic and functional characteristics may be due to impairment of their regulation (Fozza and Longinotti, 2011). Initially, the lymphocytes were most likely activated and induced to come to tumor microenvironment, as can be seen from
Figure 1 that few lymphocytes located far from tumor cells which showed nuclear expression of NFATc1 suggesting that NFATcl is essential for $\mathrm{T}$ and $\mathrm{B}$ lymphocytes activation, homeostasis and differentiation (Vaeth and Feske, 2018). Most Hodgkin tumor cells were surrounded by T-lymphocytes expressing PD-1 (Ilcus et al., 2017). The expression of PD-1 receptor driving in decreased activation of NFATCl (Sharpe and Pauken, 2018), thereby, this mechanism is one factor that was most likely led to down regulation of $N F A T C 1$ in lymphocytes surrounding tumor cells in our samples, yet the exact mechanism is still unclear. This mechanism benefits for survival of tumor cells since TILs expressing PD-1 impaired their effector functions by displaying exhausted phenotype (Thommen

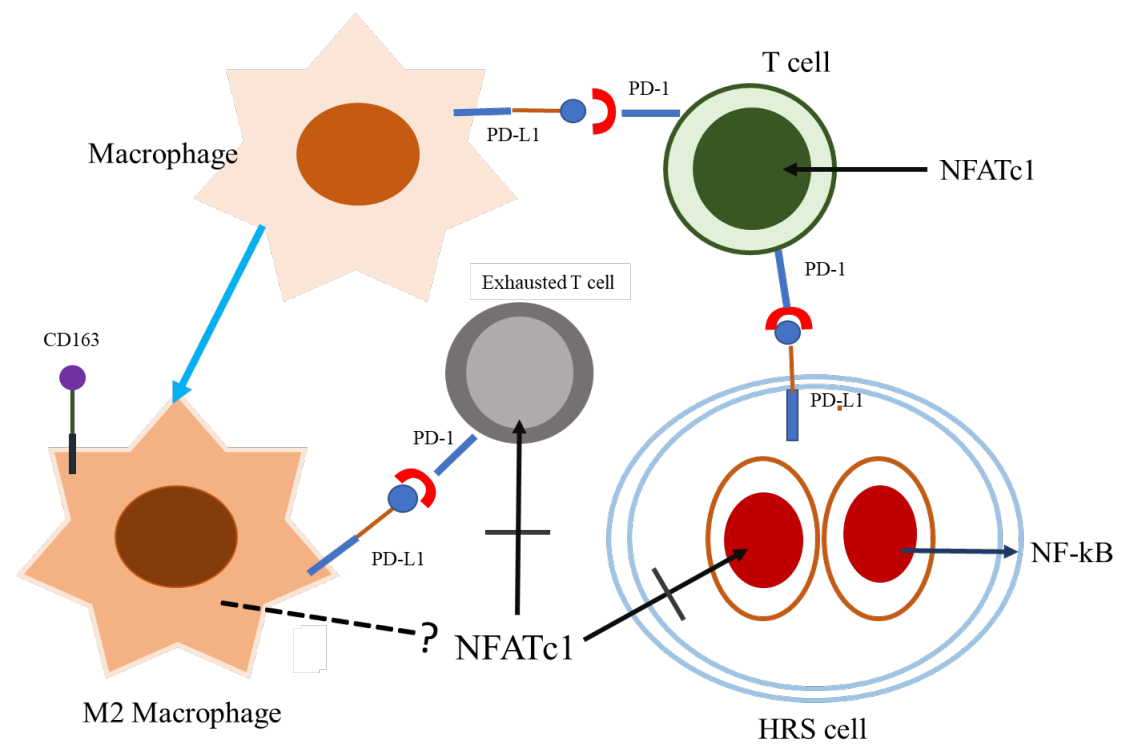

Figure 3. NFATc1 Loss in Hodgkin Cells and CTLs may Contribute to the Molecular Pathways Entailed in Tumor Microenvironment of HL. Macrophages and T cells are heavily infiltrate the background of HL. PD-L1 in macrophages inducing T cell anergy and M2 polarization. While NFATc1 is not expressed in Hodgkin cells caused by epigenetic silenced mechanism, NF- $\mathrm{KB}$ is constitutively activated in these cells. The exact regulation of this microenvironment is still unclear and need to be elucidated. 
and Schumacher, 2018; Ilcus et al., 2017). Future study is needed to unravel how the precise mechanisms control the silencing of NFATCl in tumor microenvironment of HL.

Increased TAMs CD163 + was correlated to unfavorable outcomes (Guo et al., 2016). We did not have any data of patient survival; therefore, we were unable to correlate the presence of TAMs to our patient outcomes. However, here we would like to know whether NFATc1 may have roles in activation of TAMs CD163+ in tumor microenvironment of HL. In fact, both the nuclear and cytoplasmic NFATc1 expression in TAMs CD163+ were not observed. Down regulation of NFATC1 in TAMs and Hodgkin cells may result in T cells anergy, thus, promotes tumor progression. The exact role of NFATCl in recruitment and or activation of TAM in tumor milieu is unclear

In our samples, half patients showed high density of $P D-L 1$ in tumor cells and the same percentage as in macrophages around tumor cells. Patients with high density of tumor cells expressing $P D-L 1$, also showed high density of TAMs CD163+ with $P D-L 1$ expression. This suggests TAMs have important roles in microenvironment of Hodgkin lymphoma. However, we have no information about survival data, hence, we cannot correlate the expression of $P D-L 1$ in those cells with patient survival, thus, patient prognosis. The expression of $P D-L 1$ in Hodgkin cells usually correlated to worse prognosis (Jalali et al., 2019). While the expression of $P D-L 1$ in macrophages could lead to T cell anergy and M2 polarization, indicating that high levels of $P D-L 1$ expression in macrophages were in accordance with an immunosuppressive tumor environment and decreased anti-tumor immunity (Lu et al., 2019: Jalali et al., 2019; Gordon et al., 2017). Together the expression of $P D-L 1$ in Hodgkin tumor cells and TAMs lead to worse prognosis of Hodgkin lymphoma patients (Karihtala et al., 2020). It was possible that silencing of NFATc1 expression may contribute to HRS cells to become immortal and correlated to inferior outcomes. This hypothesis should be investigated by further experiments. Understanding the exact mechanism of NFATC 1 regulation in TME could lead to development of therapeutic pathway by restoring antitumor immunity.

In conclusion NFATc1 was suppressed both in Hodgkin tumor cells and inflammatory cells surrounding the tumor cells. This condition may contribute to progressivity and aggressiveness of the diseases (Figure 3). Therefore, certain mechanisms to reactivate functional NFATCl in cHL tumor microenvironment may be necessary; hence, the tumor cells are able to be eradicated by patient's immune mechanisms.

\section{Author Contribution Statement}

All authors read, critically reviewed and approved the final manuscript. KM designed the study, analyzed all data, drafted the manuscript, conducted pathologic interpretation and lymphoma diagnosis also edited the final manuscript text. NN and NFK assisted the experimental process. IK contributed to lymphoma diagnosis. RPR and ZH contributed to the preparation of the manuscript, editing and review. This manuscript is a part of an approved student thesis

\section{Acknowledgements}

We express gratitude to all our affiliations and our teamwork for their whole collaboration and assistance.

\section{Funding Statement}

This work has been supported by Lecturer Research Grant of Faculty of Medicine, University of Sriwijaya, contract number No. 002/UN9.1.4/UPPM/PL/VII/2019, July 10th, 2019.

\section{Ethics Statement}

The study which has involved paraffin blocks of human tissues, was reviewed and approved by Health Research Review Committee of Mohammad Hoesin Central General Hospital and Faculty of Medicine University of Sriwijaya with Ethical Approval Certificate No. 325/ kepkrsmhfkunsri/2019

\section{References}

Akimzhanov, A, Krenacs L, Schlegel T, et al (2008). Epigenetic changes and suppression of the nuclear factor of activated $\mathrm{T}$ cell 1 (NFATc1) promoter in human lymphomas with defects inimmunoreceptor signaling. Am J Pathol, 172, 215-24.

Bigenwald C, Galimard JE, Quero L, et al (2017). Hodgkin lymphoma in adolescent and young adults: insights from an adult tertiary single-center cohort of 349 patients. Oncotarget, 8, 80073-82.

Bray F, Ferlay J, Soerjomataram I, et al (2018). Global cancer statistics: Globocan estimates of incidence and mortality worldwide for 36 cancers in 185 countries. CA Cancer $J$ Clin, 68, 394-424.

Calabretta E, d'Amore F, Carlo-Stello C (2019). Immune and Inflammatory cells of the tumor microenvironment represent novel therapeutic targets in classical Hodgkin lymphoma. Int J Mol Sci, 20, 5503.

Davoodzadeh Gholami M, Kardar GA, Saeedi Y, et al (2017). Exhaustion of T lymphocytes in the tumor microenvironment: Significance and effective mechanisms. Cell Immunol, 322, 1-14.

Ferlay J, Soerjomataram I, Ervik M, et al (2013). GLOBOCAN 2012 v1.0, Cancer incidence and mortality worldwide: IARC cancer base No. 11 [Internet]. Lyon, France: International Agency for Research on Cancer. Available from http:// globocan.iarc.fr.

Fozza C, Longinotti M (2010). T-cell traffic jam in Hodgkin's lymphoma: Pathogenetic and therapeutic implications. $A d v$ Hematol, 2011, 8p.

Gordon SR, Maute RL, Dulken BW (2017). PD-1 expression by tumour-associated macrophages inhibits phagocytosis and tumourimmunity. Nature, 545, 495-9.

Guo B, Cen H, Tan X, et al (2016). Meta-analysis of the prognostic and clinical value of tumor-associated macrophages in adult classical Hodgkin lymphoma. BMC Med, 14, 159.

Ilcus C, Bagacean C, Tempescu A. (2017). Immune checkpoint blockade: the role of PD-1-PD-L axis in lymphoid malignancies. Onco Targets Ther, 10, 2349-63.

Jalali S, Price-Troska, T, Bothun C, et al (2019). Reverse signaling via PD-L1 supports malignant cell growth and survival in classical Hodgkin lymphoma. Blood Cancer J, 9, 22.

Karihtala K, Leivonen SK, Brück O, et al (2020). Prognostic Asian Pacific Journal of Cancer Prevention, Vol 22 
impact of tumor-associated macrophages on survival is checkpoint dependent in classical Hodgkin lymphoma. Cancers, 12, naf15p.

Kim J, Bae JS (2016). Tumor-associated macrophages and neutrophils in tumor microenvironment. Mediators Inflamm, 2016, 6058147. Published online 2016 Feb 4. doi: $10.1155 / 2016 / 6058147$.

Li L, Zhang J, Chen J, et al (2018). B-cell receptor-mediated NFATc1 activation induces IL-10/STAT3/PD-L1 signaling in diffuse large B-cell lymphoma. Blood, 132, 1805-17.

Lu D, Ni Z, Liu X, et al (2019). Beyond T cells: Understanding the role of PD-1/PD-L1 in tumor-associated macrophages. J Immunol Res, 2019, 7p.

Marafioti T, Pozzobon M, Hansmann M-L, et al (2004). The NFATc1 transcription factor is widely expressed in white cells and translocates from the cytoplasm to the nucleus in a subset of human lymphomas. Br J Haematol, 128, 333-42.

Muhammad K, Alrefai H, Marienfeld R, et al (2014). NF-kB factors control the induction of NFATclin B lymphocytes. Eur J Immunol, 44, 3392-3402.

Oestreich KJ, Yoon H, Ahmed R, Boss JM (2008). NFATc1 Regulates PD-1 expression upon T cell activation. $J$ Immunol, 181, 4832-9.

Salati M, Cesaretti M, Macchia M, et al (2014). Epidemiological overview of Hodgkin lymphoma across the Mediterranean Basin. Mediterr J Hematol Infect Dis, 6, e2014048.

Shanbhag S, Ambinder R (2018). Hodgkin Lymphoma: a review and update on recent progress. CA Cancer J Clin, 68, 116-32.

Sharpe AH and Pauken KE (2018). The diverse functions of the PD1 inhibitory pathway. Nat Rev Immunol, 18, 153-67.

Singhal S, Stadanlick J, Annunziata MJ, et al (2019). Human tumor-associated monocytes/macrophages and their regulation of $\mathrm{T}$ cell responses in early-stage lung cancer. Sci Transl Med, 11, p. eaat1500.

Swerdlow S, Campo E, Harris N, et al (2017). WHO classification of tumours of haematopoietic and lymphoid tissues. Revised 4th ed. Lyon, France. IARC Press, pp 424-42.

Thommen DS, Schumacher TN (2018). T cell dysfunction in cancer. Cancer Cell, 33, 547-62.

Vaeth M and Feske S (2018). NFAT control of immune function: New frontiers for an abiding trooper [version 1;referees: 2 approved] F1000Research 7(F1000 Faculty Rev): 260.

Weniger MA, Küppers R (2016). NF-кB deregulation in Hodgkin lymphoma. Semin Cancer Biol, 8p. http://dx.doi. org/10.1016/j.semcancer.2016.05.001.

Zhou L, Deng Y, Li N, et al (2019). Global, regional, and national burden oflymphoma from 1990 to 2017: Estimates from the 2017 global burden of disease study. J Hematol Oncol, 12, 13p.

This work is licensed under a Creative Commons AttributionNon Commercial 4.0 International License. 